\title{
Las funciones de la clasificación en los catálogos en línea de bibliotecas universitarias argentinas
}

\author{
The functions of classification in Argentinian university library online catalogs
}

\author{
Ana María Martínez Tamayo, Leslie Bava, María Inés Kessler \\ Facultad de Humanidades y Ciencias de la Educación, Universidad Nacional de La Plata, Argentina \\ ammartitam@gmail.com
}

\begin{abstract}
Resumen
Se analizó una muestra de 91 catálogos en línea de bibliotecas de universidades públicas argentinas, con el fin de indagar sobre el uso de la clasificación para la recuperación de información. Se tomaron en cuenta cuatro funciones del catálogo: encontrar (signatura de clase como punto de acceso al registro), reunir (signatura de clase para encontrar conjuntos de registros sobre el mismo tema), obtener (signatura topográfica para localizar el documento en el estante) y navegar o explorar la estructura jerárquica del sistema de clasificación. Se encontraron 8 catálogos $(8,8 \%)$ sin clasificación, 83 catálogos $(91,2 \%)$ cumplían con la función de obtener, 18 (19,8\% con la de encontrar, $19(20,9 \%)$ con la de reunir y ninguno con la de navegar, 64 catálogos $(70,3 \%)$ sólo cumplían con una función (obtener). El sistema informático utilizado para el catálogo no fue determinante, pero si facilitaba las funciones. El sistema de clasificación más usado fue la Clasificación Decimal Universal (69 catálogos, $75,8 \%$ ). Se concluye que las ventajas de la clasificación para la recuperación de información están desaprovechadas en la mayoría de los catálogos de la muestra.
\end{abstract}

Palabras clave: Clasificaciones bibliográficas. Catálogos en línea. Bibliotecas universitarias. Argentina.

\section{Introducción}

A pesar de contar con medio siglo de historia, los catálogos en línea de las bibliotecas todavía no ofrecen a sus usuarios finales todas las ventajas que la clasificación puede proporcionarles, pues ha quedado limitada prácticamente a la asignación de una signatura topográfica para ordenar los documentos en el estante.

Karen Markey (2006), en una revisión sobre el tema de la clasificación, hace evidente cierta incoherencia en este sentido. Por un lado, se han empleado importantes recursos y esfuerzos en digitalizar los sistemas de clasificación más conocidos como el Sistema de Clasificación

\begin{abstract}
A sample of 91 online catalogs from Argentine public university libraries was analyzed, in order to investigate the use of classification. Four functions were taken into account: find (class number as an access point), gather (class number to bring together records on the same topic), obtain (class number to locate the document on shelf), and navigate or explore the hierarchical structure of the classification system. There were 8 catalogs $(8,8 \%)$ without classification; 83 catalogs $(91,2 \%)$ complied with the obtain function, 18 $(19,8 \%)$ with find function, $19(20,9 \%)$ with gather function, and no one with the navigate function. 64 catalogs $(70,3 \%)$ only complied with one function (obtain). The computer system used for the catalog was not determinant, but facilitated the compliance with the functions. The more used classification system was Universal Decimal Classification (69 catalogs, $75,8 \%)$. It is concluded that the advantages of classification for information retrieval are misused by most of the sample catalogs.
\end{abstract}

Keywords: Bibliographic classifications. Online catalogs. University libraries. Argentina.

Decimal de Dewey (SCDD), la Clasificación de la Biblioteca del Congreso (CBC) y la Clasificación Decimal Universal (CDU). También se han desarrollado formatos para la gestión de autoridades de la clasificación, como el de MARC 21 (Library of Congress, 2010) y el de UNIMARC (International Federation of Library Associations and Institutions, 2000). Sin embargo, estas herramientas han sido pensadas para apoyar a los bibliotecarios en la tarea de asignar una signatura de clase, pero no para el usuario final, que en general no obtiene beneficio alguno de la clasificación durante la búsqueda.

A pesar de lo anterior, a lo largo de los años se han desarrollado diversos proyectos para apro- 
vechar mejor la clasificación en el catálogo en línea, aunque la mayoría de ellos se limitó a su etapa experimental. Una iniciativa pionera fue la de AUDACIOUS, desarrollada por Robert R. Freeman y Pauline Cochrane en 1968, que utilizaba un método ingenioso para registrar las cadenas precoordinadas de CDU en el área de la física nuclear (Buxton, 1990; Pollit, 1997; Markey, 2006).

Otro experimento relevante fue el Proyecto SCDD (Markey y Demeyer, 1986; Drabenstott et al., 1990). Cada registro bibliográfico del catálogo en línea se enriqueció con una notación de SCDD, la designación verbal correspondiente a esa notación, la entrada del índice relativo de SCDD y la secuencia jerárquica de las notaciones en el esquema. Una vez que el usuario ingresaba su término de búsqueda, el sistema procuraba la coincidencia con las designaciones verbales y las entradas al índice relativo, además de permitir la navegación o exploración entre las clases supraordenadas y subordinadas.

Otros autores (Larson, 1991; Micco, 1994) implementaron sistemas que permitían vincular las notaciones de CBC con los encabezamientos de materia de Library of Congress. Cada notación agrupaba diversos encabezamientos de materia, según hubiesen sido asignados en el registro bibliográfico.

Uno de los proyectos que superó la etapa experimental y se encuentra actualmente en uso es el catálogo NEBIS-OPAC (antes ETHICS), desarrollado por la ETH-Bibliothek de Zurich, Suiza, desde 1983. Este catálogo se indiza con un tesauro trilingüe (inglés, francés y alemán), que se vincula con las notaciones de la CDU, de modo que el usuario final puede recuperar los registros bibliográficos por medio de descriptores y además acceder al despliegue de la estructura sistemática de CDU a través de las notaciones (Pika, 2007).

Las nuevas herramientas de descubrimiento del tipo Endeca (Endeca Technologies, Estados Unidos), que se aplican a los llamados catálogos de próxima generación o NextGen, facilitan desde la tecnología el uso de la clasificación como punto de acceso de materia (Bland y Sttofan, 2008). Un ejemplo de estos nuevos catálogos es el de las bibliotecas de la North Carolina State University de Estados Unidos. El módulo de consulta cuenta con la opción browse by call number (explore por signatura topográfica), que una vez seleccionada remite al primer nivel jerárquico del esquema de $\mathrm{CBC}$, incluyendo la notación y la designación verbal de dicha notación, por ejemplo S - Agriculture.
En el mismo sentido, el proyecto titulado Classified Catalog Browse, de la Western North Carolina Library Network de Estados Unidos, muestra el esquema completo de CBC con todos sus niveles jerárquicos. Esto se logra con un algoritmo que procura la extracción de las notaciones de los registros bibliográficos y de los registros de autoridad, con el fin de construir un despliegue en línea de las notaciones con sus designaciones verbales. El usuario final puede navegar o explorar el esquema de la clasificación, elegir una notación y ejecutar una búsqueda marcando con un clic la notación. Además puede vincular la notación con los encabezamientos de materia de Library of Congress (Bland y Stoffan, 2008).

También en la biblioteca de la Katholieke Universiteit Leuven, Bélgica, se ha evaluado una interfaz experimental para el catálogo que enriquece el acceso por materia a través de la navegación por CDU. La interfaz despliega el índice de la clasificación (notación y designación verbal) y una vez que se selecciona una notación (mediante un clic del mouse), se inicia la búsqueda en el catálogo. Permite lograr una coincidencia total entre las notaciones de CDU y los descriptores de un tesauro trilingüe, haciendo que CDU sea mucho más accesible al usuario final. Las notaciones de CDU pueden truncarse y combinarse con operadores booleanos. Además, cada notación de CDU puede situarse en su estructura jerárquica, que se genera automáticamente. La cantidad de aciertos (hits) de registros bibliográficos vinculados con cada notación se muestra al usuario final en la pantaIla (Schallier, 2005).

Por otro lado, la Declaración de Principios Internacionales de Catalogación, aprobada y publicada por la International Federation of Library Associations and Institutions (IFLA), establece entre otras las siguientes funciones para el catálogo en línea (International Federation of Library Associations and Institutions, 2009):

- Encontrar los recursos bibliográficos disponibles en la colección, por autor, título, materia $u$ otros atributos. Suele considerarse que buscar información por medio de la notación implica una gran dificultad para el usuario final, porque éste generalmente no conoce el significado de esa notación. Sin embargo, esta dificultad puede paliarse con un buen programa de formación de usuarios y una lista de autoridades de clasificación que le muestre al usuario la notación, acompañada de su correspondiente designación verbal. Así, un ingeniero civil especializado en puentes, obtendrá información valiosa de un catálogo clasificado con SCDD, cuando 
aprenda que la notación 624 es ingeniería civil y que 624.2 es justamente puentes. También le será de gran utilidad que su búsqueda por una palabra clave o un término controlado pueda limitarse a la notación 624.2.

- Reunir o encontrar conjuntos de recursos bibliográficos que comparten un mismo autor, una misma materia, etc. Desde que James Duff Brown inició la modalidad de estantería abierta al público, en la Inglaterra de fines del siglo diecinueve, la clasificación le ofreció al usuario la posibilidad de encontrar reunidos en un sólo lugar todos los documentos sobre un mismo tema, con dos importantes ventajas: a) la posibilidad de elegir más de un documento sobre la misma temática; y b) sustituir un documento faltante en la estantería por otro de similar contenido. Los catálogos en línea pueden ofrecer esas ventajas mediante opciones como navegar la estantería -browse the shelf- o simplemente haciendo un clic en la signatura de clase de un registro bibliográfico previamente seleccionado, es decir mediante un hipervínculo entre los distintos registros bibliográficos. Esta posibilidad de curiosear el estante virtual ha sido recomendada desde hace más de quince años (Koh, 1995) y se encuentra disponible en algunos catálogos en línea, por ejemplo el de Library of Congress.

- Obtener un recurso bibliográfico por medio del préstamo a domicilio o el acceso en línea al texto completo. El usuario que selecciona un recurso bibliográfico en el catálogo, puede obtener el documento para préstamo a domicilio o en sala de lectura mediante la signatura topográfica. Es la función tradicional de la clasificación y en muchos catálogos en línea la única función existente.

- Navegar o explorar el catálogo a través de las relaciones entre obras, expresiones, manifestaciones e ítems, o por otros atributos como por ejemplo la estructura de un sistema de clasificación. Muchos usuarios finales enfrentan dificultades para definir sus términos de búsqueda y armar estrategias con los operadores booleanos (Borgman, 1986, 1996; Holley, 1989; Ríos García, 1991). Por este motivo, algunos autores han hecho hincapié en la necesidad de que los catálogos en línea ofrezcan a los usuarios finales la posibilidad de explorar índices de autor, título o materia (Hildreth, 2000; Markey, 2006), pues se ha comprobado que esta búsqueda exploratoria obtiene resultados distintos y complementarios a la búsqueda por pala- bras clave. Sin embargo, el índice alfabético de materia, que generalmente se basa en descriptores o encabezamientos de materia, no ofrece la posibilidad de contextualizar un concepto dado. En cambio, los sistemas de clasificación estructuran lógicamente los conceptos, de modo tal que cada concepto en el esquema ocupa una posición definida con respecto a los demás conceptos y aparece contextualizado por sus relaciones de supraordenación y subordinación. Al navegar por la estructura sistemática, el usuario final cuenta con más posibilidades de identificar su tema de interés y ubicarlo en un dominio de conocimiento determinado (Weinberg, 1995).

Mientras que por un lado se describen las experiencias y los beneficios de la clasificación para la recuperación por materia, otros autores como Catalán Vega y Prieto Castro (1999) en España, o Slavic (2006a) en Croacia, analizaron el uso de la clasificación en catálogos en línea de distintas bibliotecas, concluyendo que la clasificación está desaprovechada en la mayoría de los catálogos.

Sobre la base de lo antes expuesto, el objetivo de este trabajo es indagar el uso que se hace de la clasificación en los catálogos en línea de bibliotecas universitarias argentinas y establecer si cumplen con las cuatro funciones mencionadas: encontrar, reunir, obtener y navegar.

\section{Metodología}

Para conformar la muestra, se identificaron 108 bibliotecas pertenecientes a las universidades nacionales de Buenos Aires, La Plata, Rosario, Córdoba, Cuyo, Nordeste, Tucumán y Patagonia, todas ellas del sector público. De estas bibliotecas, 8 fueron bibliotecas o sistemas de información centrales y 100 bibliotecas de facultades o escuelas. En total, 91 bibliotecas $(84,3 \%)$ contaban con catálogo en línea.

En cada uno de estos catálogos se analizaron las siguientes cuatro funciones:

- Encontrar: El catálogo cumplía con esta función si la notación se podía usar como elemento para la recuperación de información. En algunos catálogos era posible recuperar la notación como palabra clave, pero esta opción se descartó por presentar serios inconvenientes al recuperar también otros números. Por ejemplo, al solicitar la notación de CDU 982 (historia argentina), se obtuvieron también registros con el año de publicación 1982. Se consideró entonces que el catálogo cumplía con la función, sólo si permi- 
tía limitar la búsqueda al campo de la signatura topográfica.

- Reunir: El catálogo cumplía con esta función si permitía encontrar otros registros del mismo tema, marcando con el mouse la signatura de clase de un registro seleccionado previamente.

- Obtener: El catálogo cumplía con esta función si la notación se utilizaba para indicar la localización física del documento en el estante (signatura topográfica).

- Navegar: El catálogo cumplía con esta función si permitía navegar a través del esquema o estructura de la clasificación.

Además se indagó si el sistema informático utilizado para el catálogo en línea tenía alguna relación con el cumplimiento o no de las cuatro funciones mencionadas. Los sistemas informáticos se agruparon de la siguiente manera:

- Propios: sistemas informáticos desarrollados en la biblioteca.

- Externos gratuitos: sistemas informáticos diseñados por organizaciones ajenas a la biblioteca, que ofrecen sus desarrollos en forma gratuita.

- Comerciales nacionales: sistemas informáticos de venta comercial producidos en Argentina.

- Comerciales internacionales: sistemas informáticos de venta comercial producidos fuera del país como Symphony y Unicorn (SirsiDynix, Estados Unidos), Aleph (ExLibris, Estados Unidos), etc.

- Koha (LibLime, Estados Unidos): sistema integrado de bibliotecas que fue desarrollado en 2000 por la empresa Katipo Communication de Nueva Zelanda, pero que en la actualidad es responsabilidad de LibLime. Este sistema se ofrece como recurso open source.

Por último, también se indagó sobre la posible relación entre el sistema de clasificación utilizado en cada biblioteca y el cumplimiento de las cuatro funciones analizadas. Los datos se procesaron en una planilla de cálculo Excel (Microsoft, Corp., Estados Unidos).

\section{Resultados y discusión}

\subsection{Funciones identificadas en los catálogos}

Como era de esperar, 83 catálogos $(91,2 \%)$ cumplen con la función de obtener por medio de la clasificación. De hecho, todos los catálogos cumplen con esta función, pero 8 de ellos $(8,8 \%)$ lo hacen a través de un número de inventario o identificación y no por un sistema de clasificación.

En cambio, sólo 18 catálogos $(19,8 \%)$ cumplen con la función de encontrar y $19(20,9 \%)$ con la de reunir. Ningún catálogo cumple con la función de navegar. Estas cifras sugieren que, a excepción de la asignación de una signatura topográfica para obtener el documento, las funciones de la clasificación no revisten mayor importancia para los catálogos de la muestra.

\subsection{Combinación de funciones en un mismo catálogo}

Ninguno de los catálogos cumple con el total de las cuatro funciones analizadas. Hay 18 catálogos $(19,8 \%)$ que cumplen con tres funciones (encontrar + reunir + obtener), 1 catálogo $(1,1 \%)$ que cumple con dos funciones (reunir + obtener), 64 catálogos $(70,3 \%)$ que sólo cumplen con la función de obtener y 8 catálogos $(8,8 \%)$, como ya se mencionó, no utilizan un sistema de clasificación.

Los 18 catálogos que cumplen con tres funciones (encontrar + reunir + obtener), pertenecen a bibliotecas de una misma universidad, hecho que hace pensar en una decisión institucional al respecto. Fuera de esta universidad, prácticamente no se ha tomado en cuenta la posibilidad de aprovechar otras funciones de la clasificación, más allá de la signatura de clase para obtener el documento.

\subsection{Relación entre los sistemas informáticos y las funciones de la clasificación}

En la Tabla I se presenta la relación entre el sistema informático usado para cada catálogo y las funciones que cumple la clasificación. Ningún catálogo de la muestra utiliza sistemas informáticos internacionales de venta comercial. El único sistema informático nacional de venta comercial identificado en la muestra es Pérgamo (WalySoft Sistemas, Argentina).

Es difícil afirmar que existe una relación determinante entre el sistema informático y el cumplimiento de las funciones, pues la mayoría de los catálogos sólo cuentan con la función de obtener, sin importar qué sistema utilicen. Además, puede observarse que usando el mismo sistema informático, algunas bibliotecas cumplen con una o más de una función, indistintamente.

No obstante, algunos sistemas parecen facilitar el cumplimiento de las funciones. Por ejemplo, los 18 catálogos que cumplen con tres funcio- 
nes (encontrar + reunir + obtener) utilizan el sistema Koha, pero todos ellos pertenecen a una misma universidad, de modo que si bien el software facilita las funciones, también hay una decisión institucional al respecto. Otros cuatro catálogos que utilizan Koha desaprovechan estas posibilidades y sólo cumplen con la función de obtener.

\begin{tabular}{lllllllll}
\hline Funciones & \multicolumn{2}{c}{ Propio } & \multicolumn{2}{c}{ Externo gratuito } & \multicolumn{2}{c}{ Pérgamo } & \multicolumn{2}{c}{ Koha } \\
\hline & $\mathrm{n}$ & $\%$ & $\mathrm{n}$ & $\%$ & $\mathrm{n}$ & $\%$ & $\mathrm{n}$ & $\%$ \\
\hline Encontrar, reunir y obtener & 0 & 0 & 0 & 0 & 0 & 0 & 18 & 19,8 \\
\hline Reunir y obtener & 0 & 0 & 0 & 0 & 1 & 0 & 0 & 0 \\
\hline Obtener & 16 & 17,6 & 26 & 28,6 & 18 & 19,8 & 4 & 4,4 \\
\hline Sin clasificación & 3 & 3,3 & 5 & 5,5 & 0 & 0 & 0 & 0 \\
\hline
\end{tabular}

Tabla I. Sistemas informáticos en relación con las funciones de la clasificación, en una muestra de 91 catálogos de bibliotecas universitarias argentinas

\begin{tabular}{|c|c|c|c|c|c|c|c|c|c|c|}
\hline \multirow[t]{2}{*}{ Funciones } & \multicolumn{2}{|c|}{$C D U$} & \multicolumn{2}{|c|}{$S C D D$} & \multicolumn{2}{|c|}{ NLMC } & \multicolumn{2}{|c|}{ Propio } & \multicolumn{2}{|c|}{$S / C$} \\
\hline & $\mathrm{n}$ & $\%$ & $\mathrm{n}$ & $\%$ & $\mathrm{n}$ & $\%$ & $\mathrm{n}$ & $\%$ & $\mathrm{n}$ & $\%$ \\
\hline Encontrar, reunir y obtener & 17 & 18,7 & 0 & 0 & 0 & 0 & 1 & 1,1 & 0 & 0 \\
\hline Reunir y obtener & 1 & 1,1 & 0 & 0 & 0 & 0 & 0 & 0 & 0 & 0 \\
\hline Obtener & 51 & 56,0 & 1 & 1,1 & 3 & 3,3 & 9 & 9,9 & 8 & 8,8 \\
\hline
\end{tabular}

Tabla II. Sistemas de clasificación en relación con las funciones de la clasificación, en una muestra de 91 catálogos de bibliotecas universitarias argentinas.

Con el sistema Pérgamo, hay un catálogo que cumple con dos funciones (reunir y obtener), de manera que los otros 18 catálogos que usan este sistema sólo para la función obtener también desaprovechan la posibilidad de agregar al menos una función más.

Todos los catálogos diseñados con sistemas propios o externos gratuitos sólo cumplen con la función de obtener. Muchos de estos sistemas se basan en el software CDS/ISIS para Windows (Winisis), que la Unesco ofrece en forma gratuita. El software es eficiente y tanto en Argentina como en otros países de América Latina se han diseñado numerosas aplicaciones compatibles. Sin embargo, el diseño del catálogo (campos de la base de datos, despliegue de datos, etc.), queda sujeto a la decisión de cada biblioteca, de modo que no es posible determinar si el tratamiento de la clasificación en esos catálogos tiene que ver con un impedimento del software o con las consideraciones de cada institución.

3.4. Relación entre los sistemas de clasificación y las funciones de la clasificación

En la Tabla II se muestra la relación entre los sistemas de clasificación y las funciones de la clasificación.
Tampoco parece haber una relación directa entre el sistema de clasificación y el cumplimiento de las cuatro funciones estudiadas. CDU es el sistema utilizado por la gran mayoría de los catálogos $(75,8 \%)$, pero algunos de ellos cumplen con tres funciones, otro con dos funciones y más de dos tercios con una sola función. Con otros sistemas (SCDD y NLMC) sólo se cumple la función de obtener, pero no se puede afirmar que sea a causa del sistema de clasificación.

Llama la atención la cantidad de catálogos que utilizan códigos propios para la clasificación o que no clasifican, sino que utilizan números de inventario o de identificación para localizar un documento en el estante. Sumados, estos catálogos constituyen el $18,7 \%$ de la muestra. Se considera que este tipo de prácticas no se justifica hoy en día e impiden una correcta cooperación interbibliotecaria.

\section{Conclusiones}

La principal conclusión es el escaso aprovechamiento de las funciones de la clasificación en los catálogos en línea de la muestra, que perjudica principalmente a los usuarios finales de dichos catálogos, en particular la función de navegar o explorar el esquema jerárquico de la 
clasificación, que aparece hoy en día como la más importante para el usuario final. Esta conclusión coincide con la de otros autores (Pollit, 1997; Markey, 2006; Slavic, 2006a; Bland y Stoffan, 2008).

Algunos sistemas informáticos como Koha facilitan el tratamiento de las cuatro funciones analizadas en este trabajo, aunque también es necesario que la comunidad bibliotecaria tome las decisiones adecuadas al respecto. En este sentido, es importante tener en cuenta los nueve servicios que Slavic (2006b) recomienda para la interfaz del catálogo, respecto a la clasificación:

- Ventana para la navegación con mapas de conocimiento

- Despliegue de notaciones con sus correspondientes designaciones verbales

- Número de aciertos para cada clase

- Hipervínculos entre notaciones y referencias de véase y véase además

- Despliegue de las clases seleccionadas, con sus clases supraordenadas y subordinadas

- Diferente color para cada faceta

- Despliegue con sangría jerárquica de las designaciones verbales de las clases y puesta de relieve de la clase seleccionada en la jerarquía

- Función de cerrar/expandir una jerarquía

- Transición desde la opción de búsqueda a la navegación y viceversa.

IFLA, en sus directrices para el despliegue de datos en catálogos en línea, recomienda que si se cuenta con una lista de estructura jerárquica, como es el caso de la clasificación, el catálogo debe facilitar la navegación siguiendo esos vínculos jerárquicos, si es posible mediante un árbol de conocimiento en una interfaz gráfica (International Federation of Library Associations and Institutions, 2005).

El sistema de clasificación utilizado no tiene relación con el cumplimiento de las funciones. No obstante, la opción abrumadoramente mayoritaria por CDU hace pensar en la posibilidad de que en algún momento este sistema de clasificación pueda convertirse en una norma para las bibliotecas universitarias públicas. CDU se utiliza en 124 países, ha sido traducida a 39 idiomas y es el estándar para la red bibliotecaria de 34 países (Slavic, 2008). Además, en las últimas dos décadas, el Consorcio CDU se ha ocupado de mejorar sustancialmente este sistema de clasificación, revisando varias de sus clases, tanto principales como auxiliares, con el propósito de lograr una mayor facetización. También se encuentra desarrollando varios proyectos para su inclusión en la Web (Slavic, 2008; Slavic et al., 2008, 2009). La Asociación Española de Normalización, responsable de la edición de CDU en español, publica regularmente las nuevas ediciones y actualizaciones de este sistema de clasificación, a precios más accesibles en nuestro medio que otros sistemas (Consorcio CDU, 2000, 2004).

Por último, si en el futuro, como se ha mencionado en este trabajo, la clasificación vuelve a constituir un punto de acceso para la recuperación en el catálogo, las bibliotecas universitarias deben abandonar ciertas prácticas como la clasificación propia o la localización por números de inventario o identificación.

\section{Referencias}

Bland, Robert N.; Stoffan, Mark A. (2008). Returning classification to the catalog. // Information Technology and Libraries. 27:3 (September 2008) 55-60. http://www. ala.org/ala/mgrps/divs/lita/ital/272008/2703sep/bland_pd f.cfm (30 Ene 2011).

Borgman, Christine L. (1986). Why are online catalogs hard to use? Lessons learned from information retrieval studies. /I Journal of the American Society for Information Science. 37:6 (June, 1986) 387-400.

Borgman, Christine L. (1996). Why are online catalogs still hard to use? // Journal of the American Society for Information Science. 47:7 (July, 1996) 493-503.

Buxton, Andrew B. (1990). Computer searching of UDC numbers. // Journal of Documentation. 46:3 (March, 1990) 193-217.

Catalán Vega, Marcos Antonio; Prieto Castro, Elisa (1999). Recuperación por sistema de clasificación en los OPACS de las bibliotecas universitarias españolas. Boletín ANABAD. 49: 1, 161-173. http://dialnet.unirioja.es/ servlet/articulo?codigo $=51137$ (30 Ene 2011).

Consorcio CDU (2000). Clasificación decimal universal. Madrid: Asociación Española de Normalización (UNE 50001:2000).

Consorcio CDU (2004). Clasificación decimal universal: primera modificación. Madrid: Asociación Española de Normalización (UNE 50001/1M:2004).

Drabenstott, Karen [Markey]; Demeyer, Anh N.; Gerckens, Jeffrey; Poe, Daryl (1990). // Library Resources and Technical Services. 34 (April, 1990) 179-198.

Hildreth, Charles R. (2000). Browsing and exploring: a new paradigm for IR/OPAC system design. // Online catalog design models: are we moving in the right direction?: a report submitted to the Council on Library Resources August, 1995 [updated March 27, 2000]. [s.L]: The Author. http://myweb.cwpost.liu.edu/childret/clr-opac.html (30 Ene 2011).

Holley, Robert P. (ed.) (1989). Subject control in online catalogs. Binghamton NY: Haworth Press.

International Federation of Library Associations and Institutions (2000). Concise UNIMARC classification format. The Hague: The Federation. http://archive.ifla.org/ $\mathrm{Vl} / 3 / \mathrm{p} 1996-1 /$ concise.htm (30 Ene 2011).

International Federation of Library Associations and Institutions (2005). Guidelines for online public access catalog 
(OPAC) displays: final report. München: K.G. Saur Verlag, p. 14-25 (IFLA series on bibliographic control).

International Federation of Library Associations and Institutions (2009). Declaración de principios internacionales de catalogación. La Haya: The Federation. http://www.ifla.org/files/cataloguing/icp/icp_2009-es.pdf (30 Ene 2011).

Koh, Gertrude S. (1995). Options in classification available through modern technology. // Thomas, Alan R. (ed.). Classification: options and opportunities. Binghamton NY: Haworth Press. p. 195-212.

Larson, Ray R. (1991). Classification clustering, probabilistic information retrieval and online catalog. // Library Quarterly. 61:2 (April, 1991) 133-173.

Library of Congress (2010). MARC 21 format for classification data. Washington DC: The Library. http://www.loc.gov/marc/classification/eccdhome.html (30 Ene 2011).

Markey, Karen (2006). Forty years of classification online: final chapter or future unlimited? // Cataloging and Classification Quarterly. 42(3/4):1-63.

Markey, Karen; Demeyer, Anh N. (1986). Dewey Decimal Online Classification Project: evaluation of a library Schedule and index integrated into the subject searching capabilities of an online catalog: final report. Dublin $\mathrm{OH}$ : OCLC.

Micco, Mary; Popp, Rich (1994). Improving library subject access (ILSA): a theory of clustering based in classification. // Library Hi Tech. 12:1 (March, 1994) 55-66.

Pika, Jiri (2007). Universal Decimal Classification at the ETH-Bibliothek Zurich: a Swiss perspective. // Proceedings of the International Seminar Information Access for the Global Community, 4-5 June 2007, The Hague. The Hague: UDC Consortium, p. 229-252. http://universal decimalclassification.blogspot.com/2008/07/proceedings -of-udc-seminar-2007.html (30 Ene 2011).

Pollit, Steven (1997). The key role of classification and indexing in view-based searching. // Proceedings of the 63rd IFLA General Conference, Copenhagen, Denmark, August 31-September 5, 1997. The Hague: International
Federation of Library Associations and Institutions. http://archive.ifla.org/IV/ifla63/63polst.pdf (30 Ene 2011).

Ríos García, Yolanda (1991). Catálogos en línea de acceso público: selección bibliográfica. // Revista Española de Documentación Científica. 14:2 (Junio, 1991) 121-142.

Schallier, Wouter (2005). Subject retrieval in OPAC: a study of three interfaces. // Actas del $7^{\circ}$ Congreso de ISKOCapítulo Español, Barcelona, 6-8 de julio de 2005. Barcelona: Departament de Biblioteconomia i Documentació de la Universitat de Barcelona, p. 557-567. http://bd.ub.es/isko2005/schallier.pdf (30 Ene 2011).

Slavic, Aida (2006a). The level of exploitation of Universal Decimal Classification in library OPACs: a pilot study 2006-12 [versión abreviada en inglés]. Vjesnik Bibliotekara Hrvatski. 49:3/4 (December, 2006) 155-182. http://dlist.sir.arizona.edu/arizona/bitstream/10150/10534 6/1/UDKopac_6.pdf (30 Ene 2011).

Slavic, Aida (2006b). Interface to classification: some objectives and options. Extesions \& Corrections to the UDC, $n^{\circ}$ 28. http://www.ukrbook.net/UDC_n/st_26.pdf (30 Ene 2011).

Slavic, Aida (2008). Use of the Universal Decimal Classification: a worldwide survey. // Journal of Documentation. 64:2 (February, 2008) 211-228.

Slavic, Aida; Cordeiro, María Inês; Riesthuis, Gerhard (2009a). El desarrollo de la clasificación decimal universal: 1992-2008 y más allá. // Revista Española de Documentación Científica. 32:3 (Julio-Septiembre, 2009) 107-118. http://redc.revistas.csic.es/index.php/redc/arti cle/view/488/529 (30 Ene 2011).

Slavic, Aida; Overfield, Christ; Riesthuis, Gerhard; Pika, Jiri (2009b). Multilingual UDC summary Project: 2009 update. Extensions \& Corrections of the UDC. 31 (February 2009) 13-18. http://dlist.sir.arizona.edu/arizona/bit stream/10150/118426/1/Slavic_Overfield_Riesthuis_Pik a.pdf (30 Ene 2011).

Weinberg, Bella Hass (1995). Library classification and information retrieval thesauri: comparison and contrast. // Cataloging \& Classification Quarterly. 19:3/4 (December, 1995) 23-44. 
